\title{
Prevalence of Electrolyte Disorders during the Immediate Postoperative Intensive Care - The Experience of a Large Brazilian Center
}

\author{
Tolesani Junior 0*, Pelli Seabra MC, Berenger TV and Knibel M \\ Sao Lucas Hospital of Copacabana, Brazil
}

*Corresponding author: Oswaldo Tolesani Junior, Sao Lucas Hospital of Copacabana, Brazil, Tel: 552121374600; Email: oswaldotolesani@superig.com.br

\section{Research Article \\ Volume 4 Issue 5}

Received Date: November 25, 2019

Published Date: December 30, 2019

DOI: $10.23880 /$ accmj- 16000167

\begin{abstract}
The immediate postoperative period covers the 24 hours following any surgical intervention.In order to determine the prevalence of electrolyte disturbances in the immediate postoperative period in an intensive care unit, our team assessed the presence of electrolyte disturbances in a carefully selected group of patients. Thus, 208 patients met the selection criteria. Of these, 109 (52\%) were morbidly obese, and were in the postoperative period of bariatric surgery, which allowed the design of 2 subgroups of patients differentiated by the presence or absence of such pathology. Both subgroups of patients showed no statistically significant differences in prevalence of any of the evaluated electrolyte disturbances. The most prevalent electrolyte disorder in all patients participating in the study was hypomagnesemia, followed by hypocalcemia and hypokalemia. Despite the high prevalence of hypomagnesemia and hypocalcemia, no associated clinical complications were observed.
\end{abstract}

Keywords: Eletrolyte disturbances; Immediate Post-Operatory

\section{Introduction}

The immediate postoperative period covers the 24 hours following any surgical intervention. During this period, due to the direct and indirect action of the pathology and surgical stress, there is an increased risk of organ and system involvement, bleeding and thromboembolic phenomena, leading in many cases to the need for intensive surveillance. Few studies to date have addressed the prevalence of electrolyte disturbances during the immediate postoperative period, and yet they have only partially. The most important electrolytes in daily clinical practice are: Sodium, Bicarbonate, Potassium, Magnesium, Calcium and Phosphorus.

Electrolyte disturbances are reported as an important cause of morbidity and mortality in several clinical conditions. During surgical procedures, administration of 


\section{Anaesthesia \& Critical Care Medicine Journal}

crystalloids / colloids, medications and blood products in the operating room can potentially make these disorders more prevalent, suggesting the importance of studies addressing the prevalence of these changes and evidence of associated complications.

In 2012, Sakr Y, et al. published a study proposing an association between postoperative dysnatremia and increased morbidity and mortality in surgical patients admitted to the intensive care unit [1]. In 2013, Crestanello JA, et al. published a study proposing an association between postoperative hyponatremia and increased morbidity and mortality in patients undergoing cardiac surgery [2]. In 1989, Chernow B, et al. published studies revealing the high prevalence of hypomagnesemia in postoperative intensive care patients, and increased mortality and hypokalemia associated with severe hypomagnesemia (serum magnesium less than or equal to $1.0 \mathrm{mEq} / \mathrm{dl}$ ) [3]. In the same decade, studies confirmed the high prevalence of hypomagnesemia in patients in the immediate postoperative period of cardiac surgery [4] and in the immediate postoperative period of major gastrointestinal surgery $[5,6]$.

In 2016, Gai P, et al. published an observational study that revealed a high prevalence of hypocalcemia in patients in the immediate postoperative period of total knee arthroplasty [7]. In 1975, Martin E, et al. published a study on the effect of various crystalloid solutions on postoperative hydroelectrolytic balance [8]. In 1998, IpYam PC, et al. published a study on the prevalence of changes in urea and electrolyte levels after parenteral fluid therapy during the perioperative period [9]. The objective of this observational research is to determine the overall prevalence of electrolyte disturbances of several surgeries that required intensive care during the immediate postoperative period.

\section{Goals}

To determine in a carefully selected group of patients, during immediate postoperative intensive care, the prevalence of electrolyte disturbances involving the serum concentration of sodium, potassium, calcium (in its ionic form), magnesium, bicarbonate and phosphorus. (First 24 hours postoperatively).

During the data collection period for the study, it was observed that $52 \%$ of patients were in the immediate postoperative period of bariatric surgery due to morbid obesity. Thus, it's secondary objective was to evaluate whether, in this specific subgroup, the prevalence of electrolyte disturbances in the immediate postoperative period would be different in relation to patients undergoing other surgeries and without morbid obesity $(48 \%$ of all pacients). Additionally, patients with electrolyte disturbances were followed during the immediate postoperative period for clinical complications.

\section{Methodology}

During February 9, 2011, to December 22, 2012, every patient in the immediate postoperative period admitted in intensive care units of a large brazilian hospital located in the city of Rio de Janeiro who met the inclusion criteria of the study (Table 1) had a plasma analysis made from blood samples collected in the first 6 hours after surgery to determine serum concentrations of sodium, potassium, magnesium, ionic calcium and phosphorus.

In the hospital where the study was conducted, it's a routine in all intensive care units to take blood samples of every patient admitted after surgery. The author choosed to use restrictive selection criteria in an attempt to avoid interference from nefrological and endocrinological pathologies, and from the action of regularly used medications.

- Admission in ICU unit and blood sample collecting until 6 hours after surgery

- Absence of additional comorbidities to the pathology that indicated surgical treatment, except for morbid obesity, hepatic steatosis and gastritis.

- Absent use of any oral medications except proton pump inhibitors (only 2 patients reported use of Omeprazole)

- Absence of neuroendocrinological pathologies as a surgical indication.

Table 1: Inclusion criteria for the study.

The authors opted for dosing calcium in its ionic form, due to the great influence of serum albumin values.

Following this laboratory screening, patients with laboratory abnormalities were followed during the immediate postoperative period for possible complications during 24 hours.

The laboratory parameters of normality used in this study are shown in table 2. 


\section{Anaesthesia \& Critical Care Medicine Journal}

\begin{tabular}{|l|}
\hline Sodium: 135 to $145 \mathrm{mEq} / 1$. \\
Potassium 3.5 to5.4 mEq /1. \\
Magnesium 1.8 to $2.4 \mathrm{mg} / \mathrm{dl}$. \\
Ionic Calcium 4.5 to $5.2 \mathrm{mg} / \mathrm{dl}$ \\
Phosphorus 2.5 to $4.9 \mathrm{mg}$ / dl. \\
\hline
\end{tabular}

Table 2: Laboratory parameters of normality.

\section{Results}

\section{General}

Of the 208 patients who met the study criteria, most were between 20 and 59 years old $(94,75 \%)$, with a minimum age of 14 years and a maximum of 88 years. 109 patients were morbidly obese and underwent bariatric surgery (52.4\% of the total number of surgeries studied) (Tables $3 \& 4$ ). In our study, in patients between 10 and 59 years old, diagnosis of morbid obesity predominated, due to the large volume of bariatric surgeries performed at the hospital where the research was performed and the study selection criteria. None of the 109 morbidly obese patients in this study were older than 60 years. All surgeries that were part of the study were elective. There were no cases of morbidly obese who underwent surgery other than bariatric surgery.

\begin{tabular}{|c|c|}
\hline $0-10$ years & 0 patients \\
\hline $11-20$ years & 5 patients \\
\hline $21-30$ years & 52 patients \\
\hline $31-40$ years & 79 patients \\
\hline $41-50$ years & 41 patients \\
\hline 51-60 years & 12 patients \\
\hline $61-70$ years & 9 patients \\
\hline $71-80$ years & 9 patients \\
\hline $81-90$ years & 1 patients \\
\hline
\end{tabular}

Table 3: General age distribution of the patients.

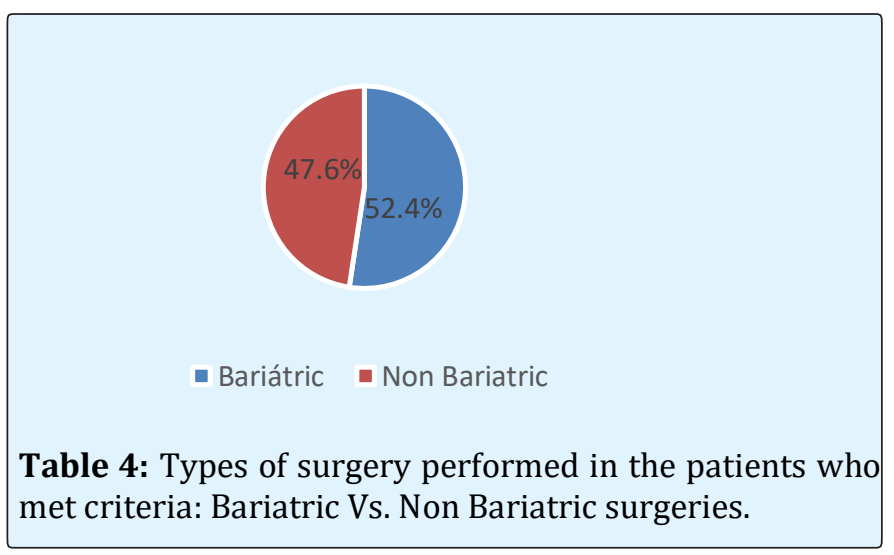

Tolesani Junior 0, et al. Prevalence of Electrolyte Disorders during the Immediate Postoperative Intensive Care - The Experience of a Large Brazilian Center. Anaesth Critic Care Med J 2019, 4(5): 000167.

\begin{tabular}{|c|c|}
\hline $0-10$ years & 0 patients \\
\hline $11-20$ years & 4 patients \\
\hline $21-30$ years & 38 patients \\
\hline $31-40$ years & 50 patients \\
\hline $41-50$ years & 15 patients \\
\hline $51-60$ years & 2 patients \\
\hline $61-70$ years & 0 patients \\
\hline $71-80$ years & 0 patients \\
\hline $81-90$ years & 0 patients \\
\hline
\end{tabular}

Table 5: Age distribution in the bariatric surgery group.

\begin{tabular}{|c|c|}
\hline $0-10$ years & 0 patients \\
\hline $11-20$ years & 1 patients \\
\hline $21-30$ years & 14 patients \\
\hline $31-40$ years & 29 patients \\
\hline $41-50$ years & 26 patients \\
\hline $51-60$ years & 10 patients \\
\hline $61-70$ years & 9 patients \\
\hline $71-80$ years & 9 patients \\
\hline $81-90$ years & 1 patients \\
\hline
\end{tabular}

Table 6: Age distribution in the non-bariatric surgery group.

\begin{tabular}{|c|c|}
\hline Type of Surgery Performed & \\
\hline Bariátric & 109 \\
\hline Neck and head & 1 \\
\hline General & 19 \\
\hline Thoracic & 10 \\
\hline Gynecological surgery & 4 \\
\hline Neurosurgery & 49 \\
\hline Orthopedic Surgery & 11 \\
\hline Otolaryngological Surgery & 1 \\
\hline Urological Surgery & 4 \\
\hline Full Surgery Number & 208 \\
\hline
\end{tabular}

Table 7: Types of surgery performed in patients with clinical criteria for the study.

Of the 99 remaining surgeries, 11 were orthopedic surgeries (10.09\%), 49 neurosurgeries (23.55\%), 10 thoracic surgeries (4.8\%), 19 non-bariatric abdominal and abdominal wall surgeries (17.59\%), 04 gynecological surgeries (3.7\%), 04 Urological surgeries (3,7\%), 01 Otolaryngological surgery $(0.48 \%), 01$ neck and head surgery $(0.48 \%)$ (Table 2$)$.

Of the total group of patients, 18 had hyponatremia (8.65\%), and 06 had hypernatremia (2.88\%). 41 patients $(19.75 \%)$ had hypokalemia, and none had hyperkalemia. 


\section{Anaesthesia \& Critical Care Medicine Journal}

151 patients (72.6\%) had hypomagnesemia, and 05 patients (2.4\%) hypermagnesemia.

In 10 of the 208 patients in the study, plasma ionic calcium was not dosed. Of the 198 patients in whom the electrolyte was dosed, 128 patients (64.65\%) had hypocalcemia, and 1 patient had hypercalcemia $(0.5 \%)$. In 8 of 208 study patients, plasma phosphate was not dosed. Of the 200 patients in whom the electrolyte was dosed, 20 patients $(10 \%)$ had hypophosphatemia. 5 patients (approximately 2.5\%) had hyperphosphatemia. During the 24-hour follow-up of patients with altered electrolyte dosages, no clinical complication that could be accredited to electrolyte disturbances of any kind was observed.

\begin{tabular}{|c|c|}
\hline Hyponatremia & $18(8,65 \%)$ \\
\hline Hypernatremia & $6(2,88 \%)$ \\
\hline Hypomagnesemia & $151(72,6 \%)$ \\
\hline Hypermagnesemia & $5(2,4 \%)$ \\
\hline Hypocalcemia & $128(64,65 \%)$ \\
\hline Hypercalcemia & $1(0,5 \%)$ \\
\hline Hypofosfatemia & $20(10 \%)^{*}$ \\
\hline Hyperfosfatemia & $5(2,5 \%)^{*}$ \\
\hline Hypokalemia & $41(19,75 \%)$ \\
\hline Hyperkalemia & $0(0 \%)$ \\
\hline
\end{tabular}

Table 8: Eletrolytic disorders found in the general group.

\section{Group of patients undergoing bariatric surgery / morbidly obese:}

Of the 109 patients in the immediate postoperative period of bariatric surgery, 9 had hyponatremia (8.26\%), and 2 had hypernatremia (1.83\%). 18 patients had hypokalemia (16.5\%). $76(69.72 \%)$ patients in the immediate postoperative period of bariatric surgery had hypomagnesemia, and 2 (1.83\%) had hypermagnesemia.

\begin{tabular}{|c|c|}
\hline Hyponatremia & $9(8,26 \%)$ \\
\hline Hypernatremia & $2(1,83 \%)$ \\
\hline Hypomagnesemia & $76(69,72 \%)$ \\
\hline Hypermagnesemia & $2(1,83 \%)$ \\
\hline Hypocalcemia & $70(69,3 \%)$ \\
\hline Hypercalcemia & $0(0 \%)$ \\
\hline Hypofosfatemia & $8(7,48 \%)^{*}$ \\
\hline Hyperfosfatemia & $3(2,8 \%)^{*}$ \\
\hline Hypokalemia & $18(16,5 \%)$ \\
\hline Hyperkalemia & $0(0 \%)$ \\
\hline
\end{tabular}

Table 9: Eletrolytic disorders found in the bariatric surgery group.
Eight patients in the immediate postoperative bariatric surgery group were not submitted to ionic calcium measurement. Of the group of 101 patients receiving this dosage, $70(69.3 \%)$ had hypocalcemia and none had hypercalcemia. 2 patients in the immediate postoperative period of bariatric surgery were not submitted to serum phosphorus measurement. Of these 107, $8(7.48 \%)$ had hypophosphatemia, and $3 \quad(2.8 \%)$ had hyperphosphatemia.

\section{Group of patients not submitted to bariatric surgery}

Of the 99 patients who did not undergo bariatric surgery, $9(9.09 \%)$ patients had hyponatremia and 4 (4.04\%) had hypernatremia. $23(23.23 \%)$ patients had hypokalemia. $75(75.76 \%)$ patients had hypomagnesemia and 3 (3.03\%) hypermagnesemia. Among non-morbidly obese patients, 6 were not submitted to serum phosphorus dosage. Of the 93 submitted to the exam, 12 $(12.90 \%)$ had hypophosphatemia and 02 patients had hyperphosphatemia (2.15\%). 2 patients in the group of non-morbidly obese patients were not submitted to serum ionic calcium dosage. Of the 97 patients evaluated, 59 (60.82\%) had hypocalcemia, and 1 (1.03\%) had hypercalcemia.

\begin{tabular}{|c|c|}
\hline Hyponatremia & $9(9,09 \%)$ \\
\hline Hypernatremia & $4(4,04 \%)$ \\
\hline Hypomagnesemia & $75(75,76 \%)$ \\
\hline Hypermagnesemia & $3(3,03 \%)$ \\
\hline Hypocalcemia & $59(60,82 \%)$ \\
\hline Hypercalcemia & $1(1,03 \%)$ \\
\hline Hypofosfatemia & $12(12,90 \%)^{*}$ \\
\hline Hyperfosfatemia & $2(2,15 \%)^{*}$ \\
\hline Hypokalemia & $23(23,23 \%)$ \\
\hline Hyperkalemia & $0(0 \%)$ \\
\hline
\end{tabular}

Table 10: Eletrolytic disorders found in the non-bariatric surgery group.

\section{Conclusion}

The most prevalent electrolyte disturbance in the total group of selected patients was hypomagnesemia, followed by hypocalcemia and hypokalemia. No cases of hyperkalaemia were observed, making it the least prevalent electrolyte disorder in the general group and in both subgroups. The second least prevalent electrolyte disorder was hypercalcemia. The researchers observed that the subgroup of non-morbidly obese patients had a higher relative prevalence of hypokalemia and 


\section{Anaesthesia \& Critical Care Medicine Journal}

hypomagnesemia, and that the morbidly obese subgroup had a higher relative prevalence of hypocalcemia, but without statistical significance ( $p$ value greater than 0.05 ). Despite the high prevalence of hypomagnesemia and hypocalcemia, no clinical complications that could be associated with these electrolyte disturbances were observed in all the patients that joined this study.

\section{References}

1. Sakr Y, Rother S, Ferreira AM, Ewald C, Dünisch P, et al. (2013) Fluctuations in serum sodium level are associated with an increased risk of death in surgical ICU patients. Crit Care Med 41(1): 133-142.

2. Crestanello JA, Phillips G, Firstenberg MS, SaiSudhakar C, Sirak J, et al. (2013) Postoperative hyponatremia predicts an increase in mortality and in-hospital complications after cardiac surgery. J Am Coll Surg 216(6): 1135-1143.

3. Chernow B, Bamberger S, Stoiko M, Vadnais M, Mills $S$, et al. (1989) Hypomagnesemia in patients in postoperative intensive care. Chest 95(2): 391-397.

4. Aglio LS, Stanford GG, Maddi R, Boyd JL Nussbaum S, et al. (1991) Hypomagnesemia is common following cardiac surgery. J Cardiothorac Vasc Anesth 5(3): 201-208.

5. Sanchez-Capuchino A, McConachie I (1994) Perioperative effect of major gastrointestinal surgery on serum magnesium. Anaesthesia 49(10): 912-914.

6. Van Gaal L, Delvigne C, Vandewoude M, Cogge E, Vaneerdeweg W, et al. (1987) Evaluation of magnesium before and after jejuno-ileal versus gastric bypass surgery for morbid obesity. J Am Coll Nutr 6(5): 397-400.

7. Gai P, Sun H, Sui L, Wang G (2016) Hypocalcaemia After Total Knee Arthroplasty and its Clinical Significance. Anticancer Res 36(3): 1309-1311.

8. Martin E, Peter K, Glocke M, Hilgenfeldt F, Kersting $\mathrm{KH}$, et al. (1975) The effect of various electrolyte solutions on the water and electrolyte balance during thepostoperative phase. Infusionsther Klin Ernahr 2(5): 338-343.

9. Ip-Yam PC, Wood PJ, Seng C (1998) Audit of changes in serum urea and electrolytes following perioperative intravenous fluid therapy. Singapore Med J 39(1): 20-24. 